\title{
On coercive variational integrals*
}

\author{
Chuei Yee Chen Jan Kristensen
}

September 11, 2016

\begin{abstract}
It is well-known that sequential weak lower semicontinuity of a variational integral

$$
\mathfrak{F}(u, \Omega)=\int_{\Omega} F(\nabla u(x)) \mathrm{d} x
$$

on the Sobolev space $\mathrm{W}^{1, p}\left(\Omega, \mathbb{R}^{N}\right)$ under a $p$-growth condition on the integrand $F$ is equivalent to quasiconvexity in the sense of Morrey. We show that coercivity on Dirichlet classes likewise is equivalent to a quasiconvexity condition. We also discuss more general notions of coercivity, and in the case of positively $p$-homogeneous integrands $F$ we establish the existence of minimizers for a class of non-coercive quasiconvex variational integrals.
\end{abstract}

\section{Introduction}

Let $F: \mathbb{R}^{N \times n} \rightarrow \mathbb{R}$ be a continuous integrand satisfying for an exponent $p \in$ $[1, \infty)$ and constant $k>0$ the growth condition

$$
|F(z)| \leq k\left(|z|^{p}+1\right)
$$

for all matrices $z \in \mathbb{R}^{N \times n}$. We consider the corresponding variational integral

$$
\mathfrak{F}(u, \Omega)=\int_{\Omega} F(\nabla u(x)) \mathrm{d} x
$$

defined for Sobolev mappings $u \in \mathrm{W}^{1, p}\left(\mathbb{R}^{n}, \mathbb{R}^{N}\right)$ and bounded open subsets $\Omega \subset \mathbb{R}^{n}$. For a mapping $g \in \mathrm{W}^{1, p}\left(\mathbb{R}^{n}, \mathbb{R}^{N}\right)$ and a non-empty bounded open subset $\Omega \subset \mathbb{R}^{n}$ we consider $\mathfrak{F}(\cdot, \Omega)$ on the Dirichlet class

$$
\mathrm{W}_{g}^{1, p}\left(\Omega, \mathbb{R}^{N}\right)=\left\{g+\varphi: \varphi \in \mathrm{W}_{0}^{1, p}\left(\Omega, \mathbb{R}^{N}\right)\right\} .
$$

We emphasize that this definition of Dirichlet class differs somewhat from the usual definition, but it is convenient for our purposes here, besides it is equivalent to the standard definition whenever $\Omega$ is a $\mathrm{W}^{1, p}$ extension domain (see [27]). It

* Dedicated to Nicola Fusco on the occasion of his 60th Anniversary 
is by now well-known (see $[14,30,28]$ and compare also [8, Remark 8.5(iii)]) that $\mathfrak{F}(\cdot, \Omega)$ is sequentially weakly lower semicontinuous on $\mathrm{W}_{g}^{1, p}\left(\Omega, \mathbb{R}^{N}\right)$ if and only if $F$ is quasiconvex (see Sect. 2 for notation and terminology). We shall generalize this semicontinuity result in the spirit of [26], see Theorem 5.1 in Section 5 . if

Fix an exponent $q \in[1, p]$. We say that $\mathfrak{F}(\cdot, \Omega)$ is $\mathrm{L}^{q}$ coercive on $\mathrm{W}_{g}^{1, p}\left(\Omega, \mathbb{R}^{N}\right)$

$$
\mathfrak{F}(u, \Omega) \rightarrow \infty \text { as }\|\nabla u\|_{q, \Omega} \rightarrow \infty \text { through } u \in \mathrm{W}_{g}^{1, p}\left(\Omega, \mathbb{R}^{N}\right) .
$$

We use this terminology also for $q=1$, which is not standard but is convenient here. The notion of $\mathrm{L}^{q}$ coercivity turns out to be a property of the integrand $F$ and thus is independent of both $\Omega$ and $g$, see Proposition 3.1.

There are various ways in which one can ensure that (1.4) holds. The most obvious one is to require that $F$ satisfies a pointwise $q$-coercivity condition: there exist constants $c_{1}, c_{2}>0$ such that $F(z) \geq c_{1}|z|^{q}-c_{2}$ holds for all $z \in \mathbb{R}^{N \times n}$. This however is unnecessarily restrictive in the multi-dimensional vectorial case $n, N \geq 2$ and not satisfied in many interesting cases (see [5] and also Proposition 2.1 below). Instead a more natural condition ensuring (1.4) is that of $\mathrm{L}^{q}$ mean coercivity: there exist constants $c_{1}>0, c_{2} \in \mathbb{R}$ such that

$$
\mathfrak{F}(u, \Omega) \geq c_{1}\|\nabla u\|_{q, \Omega}^{q}+c_{2}
$$

for all $u \in \mathrm{W}_{g}^{1, p}\left(\Omega, \mathbb{R}^{N}\right)$. It might be a little surprising that the two conditions (1.4) and (1.5) are in fact equivalent under our assumptions. This and the fact that they in turn are equivalent to a quasiconvexity condition is our first result:

Proposition 1.1. Let $F: \mathbb{R}^{N \times n} \rightarrow \mathbb{R}$ be a continuous integrand satisfying for an exponent $p \in[1, \infty)$ and constant $k>0$ the growth condition (1.1). Then for an exponent $q \in[1, p]$ the following five statements are mutually equivalent:

(i) $F$ is $\mathrm{L}^{q}$ coercive: the condition (1.4) holds for any choice of non-empty, bounded open subset $\Omega \subset \mathbb{R}^{n}$ and any boundary datum $g \in \mathrm{W}^{1, p}\left(\mathbb{R}^{n}, \mathbb{R}^{N}\right)$.

(ii) There exist a non-empty bounded open subset $\Omega \subset \mathbb{R}^{n}$ and a boundary datum $g \in \mathrm{W}^{1, p}\left(\mathbb{R}^{n}, \mathbb{R}^{N}\right)$ such that condition (1.4) holds.

(iii) $F$ is $\mathrm{L}^{q}$ mean coercive: the condition (1.5) holds for any choice of nonempty, bounded open subset $\Omega \subset \mathbb{R}^{n}$ and any boundary datum $g \in \mathrm{W}^{1, p}\left(\mathbb{R}^{n}, \mathbb{R}^{N}\right)$.

(iv) There exist a non-empty bounded open subset $\Omega \subset \mathbb{R}^{n}$ and a boundary datum $g \in \mathrm{W}^{1, p}\left(\mathbb{R}^{n}, \mathbb{R}^{N}\right)$ such that condition (1.5) holds.

(v) There exist a constant $c>0$ and a matrix $z_{0} \in \mathbb{R}^{N \times n}$ such that the integrand $z \mapsto F(z)-c|z|^{q}$ is quasiconvex at $z_{0}$.

We present the elementary proof of Proposition 1.1 in Section 3.

It is not difficult to see that a continuous integrand $F: \mathbb{R}^{N \times n} \rightarrow \mathbb{R}$ which is positively $p$-homogeneous, that is, $F(t z)=t^{p} F(z)$ holds for all $z \in \mathbb{R}^{N \times n}$ and $t \geq 0$, is $\mathrm{L}^{q}$ coercive precisely when there exists a constant $c>0$ such that

$$
\int_{\Omega} F(\nabla \varphi(x)) \mathrm{d} x \geq c\|\nabla \varphi\|_{q, \Omega}^{\frac{p}{q}}
$$


holds for all $\varphi \in \mathrm{W}_{0}^{1, p}\left(\Omega, \mathbb{R}^{N}\right)$. Here we can take $\Omega=(0,1)^{n}$ or any other non-empty bounded open subset of $\mathbb{R}^{n}$. Following [16] we provide in Section 2 non-trivial examples of integrands $F$ satisfying (1.6) for $q=p$. We recall that [32] gave an example of a nonnegative $\frac{n}{2}$-homogeneous quasiconvex integrand on $\mathbb{R}^{n \times n}$ for even dimensions $n \geq 4$ which is $\mathrm{L}^{q}$ coercive for each $q<\frac{n}{2}$ but not $\mathrm{L}^{\frac{n}{2}}$ coercive. (In fact, in an obvious extension of our terminology the integrand is $\mathrm{L}^{\frac{n}{2}, \infty}$ coercive, where $\mathrm{L}^{\frac{n}{2}, \infty}$ is the corresponding Marcinkiewicz space.) However, it remains unclear to us if, for an exponent $q_{o} \in(1, p)$, there can exist $p$-homogeneous integrands that are $\mathrm{L}^{q}$ coercive for each $q<q_{o}$ but not for $q \in\left[q_{o}, p\right]$.

\section{Theorem 1.1.}

\section{Preliminaries}

In this paper we follow the usual convention and denote by $c$ a general constant that may vary on different occasions, even within the same line of estimates. Relevant dependencies on parameters and special constants will be suitably emphasized using parentheses or subscripts. All the norms we use on $\mathbb{R}^{n}, \mathbb{R}^{N}$ and $\mathbb{R}^{N \times n}$ will be the standard euclidean ones and denoted by $|\cdot|$ in all cases. In particular, for matrices $z, w \in \mathbb{R}^{N \times n}$ we write $z \cdot w=\langle z, w\rangle:=\operatorname{trace}\left(z^{T} w\right)$ for the usual inner product of $z$ and $w$, and $|z|:=\langle z, z\rangle^{\frac{1}{2}}$ for the corresponding euclidean norm. When $a \in \mathbb{R}^{N}$ and $b \in \mathbb{R}^{n}$ we write $a \otimes b \in \mathbb{R}^{N \times n}$ for the tensor product defined as the matrix that has the element $a_{r} b_{s}$ in its $\mathrm{r}$-th row and s-th column. Observe that $(a \otimes b) x=(b \cdot x) a$ for $x \in \mathbb{R}^{n}$, and $|a \otimes b|=|a||b|$.

Our references for function spaces and measure theory are $[2,11,17,27$, $39]$ and for calculus of variations [2, 8, 31], and the reader can find further background and explanations there if required.

For $z \in \mathbb{R}^{N \times n}$ and a non-empty, bounded open subset $\Omega \subset \mathbb{R}^{n}$ we define a class of special gradient distributions with centre of mass at $z$ :

$$
G_{z}=G_{z}^{\Omega}=\left\{(z+\nabla \varphi)_{\#}\left(\frac{\mathcal{L}^{n}\lfloor\Omega}{\mathcal{L}^{n}(\Omega)}\right): \begin{array}{c}
\varphi: \mathbb{R}^{n} \rightarrow \mathbb{R}^{N} \text { Lipschitz and } \\
\text { compactly supported in } \Omega
\end{array}\right\} .
$$

Lemma 2.1. $G_{z}$ is independent of the set $\Omega$ : if $\omega \subset \mathbb{R}^{n}$ is another non-empty, bounded open subset, then $G_{z}^{\omega}=G_{z}^{\Omega}$.

Proof. This is done by a standard exhaustion argument. Without loss of generality we assume that $z=0$ and fix a Lipschitz map $\varphi: \mathbb{R}^{n} \rightarrow \mathbb{R}^{N}$ with compact suport contained in $\Omega$. Select $\Omega^{\prime} \Subset \Omega$ such that $\varphi \equiv 0$ off $\Omega^{\prime}$ and $\mathcal{L}^{n}\left(\partial \Omega^{\prime}\right)=0$. Take $\omega^{\prime} \Subset \omega$ with $\mathcal{L}^{n}\left(\omega^{\prime}\right)=\frac{\mathcal{L}^{n}\left(\Omega^{\prime}\right)}{\mathcal{L}^{n}(\Omega)} \mathcal{L}^{n}(\Omega)$ and $\mathcal{L}^{n}\left(\partial \omega^{\prime}\right)=0$. Write

$$
\omega^{\prime}=M \cup \bigcup_{j \in J}\left(x_{j}+r_{j} \overline{\Omega^{\prime}}\right)
$$

as a disjoint union, where $\mathcal{L}^{n}(M)=0$. Define for each $x \in \mathbb{R}^{n}$,

$$
\psi(x)=\sum_{j \in J} r_{j} \varphi\left(\frac{x-x_{j}}{r_{j}}\right) .
$$


Then for instance by checking the absolute convergence of the defining series in $W_{0}^{1,1}$ we conclude that $\psi \in \mathrm{W}_{0}^{1,1}\left(\omega^{\prime}, \mathbb{R}^{N}\right)$, whereas clearly $\psi=0$ almost everywhere off $\omega^{\prime}$. Next, for almost all $x$ we have

$$
\nabla \psi(x)=\sum_{j \in J} \nabla \varphi\left(\frac{x-x_{j}}{r_{j}}\right)
$$

so in particular $|\nabla \psi(x)| \leq \operatorname{Lip}(\varphi)$ and $\psi$ is Lipschitz on $\mathbb{R}^{n}$. Clearly $\psi$ is supported in $\overline{\omega^{\prime}}$ and for $\Phi \in \mathrm{C}_{0}\left(\mathbb{R}^{N \times n}\right)$ we calculate using the choice of $\omega^{\prime}$ that

$$
f_{\omega} \Phi(\nabla \psi) \mathrm{d} x=f_{\Omega} \Phi(\nabla \varphi) \mathrm{d} x
$$

We state the definition of quasiconvexity in terms of the gradient distributions in $G_{z}$ :

Definition 2.1. A continuous integrand $F: \mathbb{R}^{N \times n} \rightarrow \mathbb{R}$ is said to be quasiconvex at $z \in \mathbb{R}^{N \times n}$ if

$$
F(z) \leq \int_{\mathbb{R}^{N \times n}} F \mathrm{~d} \mu
$$

for every $\mu \in G_{z}$. The integrand $F$ is quasiconvex if it is quasiconvex at every $z \in \mathbb{R}^{N \times n}$

It is not difficult to check that this definition is equivalent to Morrey's original definition in [30]. In the presence of the growth condition (1.1) one can strengthen the quasiconvexity inequality (2.1) and show by a routine approximation argument that if $F$ is quasiconvex at a matrix $z \in \mathbb{R}^{N \times n}$, then

$$
f_{\Omega} F(z+\nabla \varphi) \mathrm{d} x \geq F(z)
$$

holds for any non-empty bounded and open subset $\Omega$ of $\mathbb{R}^{n}$ and any mapping $\varphi \in \mathrm{W}_{0}^{1, p}\left(\Omega, \mathbb{R}^{N}\right)$.

On several occasions we shall also require the quasiconvex envelope. Recall (see $[7,8]$ ) that for a continuous integrand $F$ its quasiconvex envelope, $F^{\mathrm{qc}}$, is by definition

$$
F^{\mathrm{qc}}(z):=\sup \{G(z): G \leq F \text { and } G \text { quasiconvex }\} .
$$

It follows that either $F^{\mathrm{qc}} \equiv-\infty$ on $\mathbb{R}^{N \times n}$ or $F^{\mathrm{qc}}$ is real-valued everywhere. In the latter case it is then not difficult to see that $F^{\mathrm{qc}}$ is quasiconvex and so in particular is locally Lipschitz (see [8]). The following formula for the quasiconvex envelope is due to Dacorogna and is frequently useful.

Lemma 2.2. Let $F: \mathbb{R}^{N \times n} \rightarrow \mathbb{R}$ be a continuous integrand. Then for each $z \in \mathbb{R}^{N \times n}$ the quasiconvex envelope is given by

$$
F^{\mathrm{qc}}(z)=\inf \left\{\int_{\mathbb{R}^{N \times n}} F \mathrm{~d} \mu: \mu \in G_{z}\right\} .
$$


See $[7,8]$ and, for the general case where the integrand admits no lower bound, see the appendix of [20]. When $F$ satisfies the growth condition (1.1) and $F^{\mathrm{qc}}$ is real-valued, then it is not difficult to show that $F^{\text {qc }}$ also satisfies (1.1), though possibly with a larger constant $k$. See for instance [23, Lemma 2.5].

It is a convenient fact that quasiconvex integrands $F$ are rank one convex (see $[2,8,31]): F\left((1-\lambda) z_{0}+\lambda z_{1}\right) \leq(1-\lambda) F\left(z_{0}\right)+\lambda F\left(z_{1}\right)$ for all matrices $z_{0}$, $z_{1} \in \mathbb{R}^{N \times n}$ with $\operatorname{rank}\left(z_{1}-z_{0}\right)=1$ and all $\lambda \in(0,1)$. The following result is well-known and allows us to get pointwise bounds from integral bounds.

Lemma 2.3. If $F: \mathbb{R}^{N \times n} \rightarrow \mathbb{R}$ is rank one convex, then there exists $z_{0} \in \mathbb{R}^{N \times n}$ such that

$$
F(z) \geq F(0)+z_{0} \cdot z
$$

holds for all $z$ of rank one.

Proof. Assume first that $F$ is differentiable at 0 . In this case we are forced to take $z_{0}=F^{\prime}(0)$. In turn, we can use convexity of the function $\mathbb{R} \ni t \mapsto F(t z)$ for $z$ of rank one to deduce that $F(z) \geq F(0)+z_{0} \cdot z$ as required. In the general case we take a standard smooth mollifier $\left(\Phi_{\varepsilon}\right)_{\varepsilon>0}$ on $\mathbb{R}^{N \times n}$ and put $F_{\varepsilon}=\Phi_{\varepsilon} * F$. Then $F_{\varepsilon}$ is smooth and rank one convex, so by the above argument we have $F_{\varepsilon}(z) \geq F_{\varepsilon}(0)+F_{\varepsilon}^{\prime}(0) \cdot z$ for $z$ of rank one. Because $F$ as a real-valueed rank one convex function must be locally Lipschitz we have that $F_{\varepsilon}$ converges locally uniformly to $F$ and that $\left(F_{\varepsilon}^{\prime}(0)\right)_{\varepsilon \in(0,1)}$ is bounded in $\mathbb{R}^{N \times n}$. Consequently, any limit point for $F_{\varepsilon}^{\prime}(0)$ as $\varepsilon \searrow 0$ will do as $z_{0}$.

Proposition 2.1. Let $F, G: \mathbb{R}^{N \times n} \rightarrow \mathbb{R}$ be two continuous integrands satisfying (1.1) for $a 1 \leq p<\infty$. Assume that

$$
\int_{\Omega} F(\nabla \varphi) \mathrm{d} x \geq \int_{\Omega} G(\nabla \varphi) \mathrm{d} x
$$

holds for all $\varphi \in \mathrm{W}_{0}^{1, p}\left(\Omega, \mathbb{R}^{N}\right)$. Then there exists $z_{0} \in \mathbb{R}^{N \times n}$ such that

$$
F(z) \geq G(z)+z_{0} \cdot z
$$

for all $z \in \mathbb{R}^{N \times n}$ with $\operatorname{rank}(z) \leq 1$.

This is essentially in [5], see also [3] for the case $p=1$. However, when $p=1$ one can say much more and this is the contents of Lemma 4.2 below, that in turn follows from [21]. We deduce Proposition 2.1 from Lemmas 2.2, 2.3 and the fact that rank one convexity is a necessary condition for quasiconvexity.

Proof. Put $H(z)=F(z)-G(z)$ for $z \in \mathbb{R}^{N \times n}$. The assumption and Lemma 2.2 yields $H^{\mathrm{qc}}(0) \geq 0$, and hence that $H^{\mathrm{qc}}$ is real-valued and quasiconvex. But then $H^{\mathrm{qc}}$ is also rank one convex, so by Lemma 2.3 we find $z_{0} \in \mathbb{R}^{N \times n}$ such that

$$
H(z) \geq H^{\mathrm{qc}}(z) \geq H^{\mathrm{qc}}(0)+z_{0} \cdot z \geq z_{0} \cdot z
$$

for all $z$ of rank one. This is the assertion. 
A closely related result concerns invariant integrals. We state it for comparison, but emphasize that it is in fact a consequence of more general results found in the literature, see [8] and the references given there.

Lemma 2.4. Let $F, G: \mathbb{R}^{N \times n} \rightarrow \mathbb{R}$ be two continuous integrands satisfying (1.1). Assume that for some non-empty, bounded and open subset $\Omega \subset \mathbb{R}^{n}$ and a mapping $g \in \mathrm{W}^{1, p}\left(\mathbb{R}^{n}, \mathbb{R}^{N}\right)$ we have

$$
\int_{\Omega} F(\nabla u) \mathrm{d} x=\int_{\Omega} G(\nabla u) \mathrm{d} x
$$

for all $u \in \mathrm{W}_{g}^{1, p}\left(\Omega, \mathbb{R}^{N}\right)$. Then $F=G+\mathcal{N}$, where $\mathcal{N}$ is a null Lagrangian satisfying (1.1) (so $\mathcal{N}(z)$ is an affine function of minors of $z$ of order at most p).

Proof. Put $H=F-G$. Assume first that $H$ is smooth. For any $u \in \mathrm{W}_{g}^{1, p}\left(\Omega, \mathbb{R}^{N}\right)$ and $\varphi \in \mathrm{W}_{0}^{1, p}\left(\Omega, \mathbb{R}^{N}\right)$ we have

$$
\int_{\Omega} H(\nabla u+t \nabla \varphi) \mathrm{d} x=0 \quad \forall t \in \mathbb{R} .
$$

Fix $z \in \mathbb{R}^{N \times n}$. Take $B_{r}\left(x_{0}\right) \Subset \Omega$ and a standard cut-off function: $\rho \in \mathrm{W}^{1, \infty}(\Omega)$ and $\mathbf{1}_{B_{r}\left(x_{0}\right)} \leq \rho \leq \mathbf{1}_{\Omega}$. Take $u(x)=(1-\rho(x)) g(x)+\rho(x) z\left(x-x_{0}\right)$ and let $\varphi \in \mathrm{W}_{0}^{1, \infty}\left(B_{r}\left(x_{0}\right), \mathbb{R}^{N}\right)$. We extend $\varphi$ by $0 \in \mathbb{R}^{N}$ off $B_{r}\left(x_{0}\right)$ and obtain then by differentiation of (2.2) twice at $t=0$ that

$$
\int_{B_{r}\left(x_{0}\right)} H^{\prime \prime}(z)[\nabla \varphi, \nabla \varphi] \mathrm{d} x=0 .
$$

Consequently, the quadratic form $H^{\prime \prime}(z)$ is quasi-affine, and hence rank one affine for all $z$. But then $H$ is rank one affine, and therefore must be an affine function of the minors (see [8, Theorem 5.20]). That the minors must all be of order at most $p$ follows easily from (1.1). The general case is dealt with by mollification as in the proof of Lemma 2.3.

\section{Characterization of coercivity}

In this section we give the proof of Proposition 1.1, and in the process of doing that we also collect a few elementary observations on coercivity. But let us start by displaying our definition of coercivity.

Definition 3.1. Let $1 \leq q \leq p<\infty$ and $F: \mathbb{R}^{N \times n} \rightarrow \mathbb{R}$ be a continuous integrand satisfying the growth condition (1.1). We say that $F$ is $\mathrm{L}^{q}$ coercive if for all non-empty, bounded and open $\Omega \subset \mathbb{R}^{n}$ and any $g \in \mathrm{W}^{1, p}\left(\Omega, \mathbb{R}^{N}\right)$ we have

$$
\int_{\Omega} F(\nabla u(x)) \mathrm{d} x \rightarrow \infty \text { as }\|\nabla u\|_{q, \Omega} \rightarrow \infty \text { through } u \in \mathrm{W}_{g}^{1, p}\left(\Omega, \mathbb{R}^{N}\right) .
$$


As already mentioned in the introduction there is a natural variant of the coercivity condition involving the Marcinkiewicz space $\mathrm{L}^{p, \infty}$ that turn up in a concrete example. The definition is the obvious one where one replaces the $\mathrm{L}^{q}$ norm in Definition 3.1 by the $\mathrm{L}^{p, \infty}$-quasinorm:

$$
\|f\|_{p, \infty, \Omega}=\sup _{t>0}\left(t \mathcal{L}^{n}(\{x \in \Omega:|f(x)|>t\})^{\frac{1}{p}}\right) .
$$

Most of the results that we prove for $\mathrm{L}^{q}$ coercivity in fact carry over to $\mathrm{L}^{p, \infty}$ coercivity, but here we will refrain from stating these results explicitly and instead focus the exposition on the former.

In order to verify the $\mathrm{L}^{q}$ coercivity condition it suffices to do so for any convenient choice of $\Omega, g$ :

Proposition 3.1. Let $1 \leq q \leq p<\infty$ and $F: \mathbb{R}^{N \times n} \rightarrow \mathbb{R}$ be a continuous integrand satisfying the growth condition (1.1). Let $\Omega_{0} \subset \mathbb{R}^{n}$ be a non-empty, bounded and open subset and $g_{0} \in \mathrm{W}^{1, p}\left(\mathbb{R}^{n}, \mathbb{R}^{N}\right)$. Then $F$ is $\mathrm{L}^{q}$ coercive if and only if

$$
\int_{\Omega_{0}} F(\nabla v(x)) \mathrm{d} x \rightarrow \infty \text { as }\|\nabla v\|_{q, \Omega_{0}} \rightarrow \infty \text { through } v \in \mathrm{W}_{g_{0}}^{1, p}\left(\Omega_{0}, \mathbb{R}^{N}\right) .
$$

Proof. This is straight forward. Assume the condition holds for the given $\Omega_{0}$, $g_{0}$, and let $\Omega \subset \mathbb{R}^{n}$ be non-empty, bounded and open and $g \in \mathrm{W}^{1, p}\left(\Omega, \mathbb{R}^{N}\right)$. Take $B_{2 r}\left(x_{0}\right) \Subset \Omega_{0}$ and $\phi \in \mathrm{W}_{0}^{1, \infty}\left(B_{2 r}\left(x_{0}\right)\right)$ with $\mathbf{1}_{B_{r}\left(x_{0}\right)} \leq \phi \leq \mathbf{1}_{B_{2 r}\left(x_{0}\right)}$, and likewise take $B_{R}(0) \ni \Omega$ and $\rho \in \mathrm{W}_{0}^{1, \infty}\left(B_{R}(0)\right)$ with $\mathbf{1}_{\Omega} \leq \rho \leq \mathbf{1}_{B_{R}(0)}$. Now for $u \in \mathrm{W}_{g}^{1, p}\left(\Omega, \mathbb{R}^{N}\right)$ we consider $u \in \mathrm{W}^{1, p}\left(\mathbb{R}^{n}, \mathbb{R}^{N}\right)$ with the extension $u=g$ off $\Omega$ (which is possible by our definition of Dirichlet class). Put $\tilde{w}=\rho u$ and $w(x)=\tilde{w}(R x) / R, x \in \mathbb{R}^{n}$, so that $\left.w\right|_{B_{1}(0)} \in \mathrm{W}_{0}^{1, p}\left(B_{1}(0), \mathbb{R}^{N}\right)$. Next, let

$$
v(x)=(1-\phi(x)) g_{0}(x)+r w\left(\frac{x-x_{0}}{r}\right), x \in \Omega_{0} .
$$

Then $v \in \mathrm{W}_{g_{0}}^{1, p}\left(\Omega_{0}, \mathbb{R}^{N}\right)$ and by calculation we find

$$
\begin{aligned}
\|\nabla v\|_{q, \Omega_{0}}^{q}= & \int_{\Omega_{0} \backslash B_{r}\left(x_{0}\right)}\left|\nabla\left((1-\phi) g_{0}\right)\right|^{q} \mathrm{~d} x+\left(\frac{r}{R}\right)^{n} \int_{B_{R}(0) \backslash \Omega}|\nabla(\rho g)|^{q} \\
& +\left(\frac{r}{R}\right)^{n}\|\nabla u\|_{q, \Omega}^{q}
\end{aligned}
$$

and

$$
\begin{aligned}
\int_{\Omega_{0}} F(\nabla v) \mathrm{d} x= & \int_{\Omega_{0} \backslash B_{r}\left(x_{0}\right)} F\left(\nabla\left((1-\phi) g_{0}\right)\right) \mathrm{d} x+\left(\frac{r}{R}\right)^{n} \int_{B_{R} \backslash \Omega} F(\nabla(\rho g)) \mathrm{d} x \\
& +\left(\frac{r}{R}\right)^{n} \int_{\Omega} F(\nabla u) \mathrm{d} x .
\end{aligned}
$$

It follows that $F$ is $\mathrm{L}^{q}$ coercive.

In view of the above observation, that coercivity is independent of the data $\Omega$, $g$, we may conveniently express it through the following auxiliary function. 
Definition 3.2. Let $F: \mathbb{R}^{N \times n} \rightarrow \mathbb{R}$ be a continuous integrand satisfying the growth condition (1.1). For $q \in[1, p]$ and a non-empty bounded and open subset $\Omega \subset \mathbb{R}^{n}$ we define for each $t \geq 0$,

$$
\Theta(t)=\Theta_{q}^{\Omega}(t)=\inf \left\{f_{\Omega} F(\nabla \varphi) \mathrm{d} x: \varphi \in \mathrm{W}_{0}^{1, p}\left(\Omega, \mathbb{R}^{N}\right), f_{\Omega}|\nabla \varphi|^{q} \mathrm{~d} x \geq t\right\} .
$$

Clearly $\Theta:[0, \infty) \rightarrow \mathbb{R} \cup\{-\infty\}$ is non-decreasing. Under the given assumptions it is also independent of the subset $\Omega$, and in fact it only depends on the quasiconvex envelope of the integrand:

Lemma 3.1. Let $\omega, \Omega \subset \mathbb{R}^{n}$ be non-empty bounded open subsets, and define the corresponding auxiliary function $\Theta_{q}^{\omega}$ as above. Define also for each $t \geq 0$,

$$
\Theta^{\mathrm{qc}}(t)=\inf \left\{f_{\Omega} F^{\mathrm{qc}}(\nabla \varphi) \mathrm{d} x: \varphi \in \mathrm{W}_{0}^{1, p}\left(\Omega, \mathbb{R}^{N}\right), f_{\Omega}|\nabla \varphi|^{q} \mathrm{~d} x \geq t\right\}
$$

which is of course just $\Theta_{q}^{\Omega}(t)$ for the quasiconvex envelope of $F$. Then $\Theta_{q}^{\omega}=\Theta^{\mathrm{qc}}$.

In particular, $F$ is $\mathrm{L}^{q}$ coercive if and only if its quasiconvex envelope $F^{\mathrm{qc}}$ is $\mathrm{L}^{q}$ coercive. Furthermore, we always have $\Theta(t) \geq F^{\mathrm{qc}}(0)$ for all $t \geq 0$ and equality holds at $t=0$.

Proof. From Lemma 2.2 we infer that $\Theta_{q}^{\omega}(0)=F^{\mathrm{qc}}(0)=\Theta^{\mathrm{qc}}(0)$, and so only the case $t>0$ remains. If $F^{\mathrm{qc}} \equiv-\infty$, then an argument based on Lemma 2.2 again shows that $\Theta_{q}^{\omega}(t)=-\infty$. Since, in this situation, clearly also $\Theta^{\mathrm{qc}}(t)=-\infty$ we can in the remainder of the proof focus on the case where $t>0$ and $F^{\mathrm{qc}}$ is real-valued. Let $\varepsilon>0$ and take $\varphi \in \mathrm{C}_{c}^{\infty}\left(\Omega, \mathbb{R}^{N}\right)$ with

$$
f_{\Omega}|\nabla \varphi|^{q} \mathrm{~d} x \geq t \text { and } \Theta^{\mathrm{qc}}(t)+\varepsilon>f_{\Omega} F^{\mathrm{qc}}(\nabla \varphi) \mathrm{d} x .
$$

Because $F^{\mathrm{qc}}$ is locally Lipschitz continuous and $\varphi$ is smooth we can use Lagrange interpolation on a suitably fine uniform and regular triangulation $\tau$ of $\mathbb{R}^{n}$ to find a piecewise affine Lipschitz mapping $a: \mathbb{R}^{n} \rightarrow \mathbb{R}^{N}$, which is compactly supported in $\Omega$,

$$
f_{\Omega}|\nabla a|^{q} \mathrm{~d} x \geq t \text { and } f_{\Omega} F^{\mathrm{qc}}(\nabla \varphi) \mathrm{d} x>f_{\Omega} F^{\mathrm{qc}}(\nabla a) \mathrm{d} x-\varepsilon .
$$

More precisely, first we select $\sigma>1$ close to 1 so that

$$
f_{\Omega} F^{\mathrm{qc}}(\nabla \varphi) \mathrm{d} x>f_{\Omega} F^{\mathrm{qc}}(\sigma \nabla \varphi) \mathrm{d} x-\varepsilon / 2
$$

Next, select $\delta \in\left(0,(\sigma-1) t^{\frac{1}{q}}\right]$ and a suitably fine uniform and regular triangulation $\tau$ of $\mathbb{R}^{n}$ such that the Lagrange interpolant $a$ for $\sigma \varphi$ on $\tau$ satisfies

$$
f_{\Omega}|\sigma \nabla \varphi-\nabla a|^{p} \mathrm{~d} x<\delta^{p}
$$


and

$$
f_{\Omega} F^{\mathrm{qc}}(\nabla \varphi) \mathrm{d} x>f_{\Omega} F^{\mathrm{qc}}(\nabla a) \mathrm{d} x-\varepsilon .
$$

The imposed upper bound on $\delta$ yields in a routine manner

$$
f_{\Omega}|\nabla a|^{q} \mathrm{~d} x \geq t .
$$

Finally, to ensure that $a$ is compactly supported in $\Omega$ we might have to refine the triangulation. Indeed, since the distance from the support of $\varphi$ to the boundary of $\Omega$ is strictly positive we obtain by a suitable refinement of $\tau$ that each simplex $T \in \tau$ with $T \cap \operatorname{spt}(\varphi) \neq \emptyset$ has closure $\bar{T} \subset \Omega$. Put $\tau_{\Omega}=\{T \in \tau: T \cap \operatorname{spt}(\varphi) \neq \emptyset\}$, and note that $a$ is supported in the closure of $\bigcup \tau_{\Omega}$, which in turn is compact and contained in $\Omega$.

Having established the existence of the piecewise affine approximating mapping $a$ we proceed using Lemma 2.2 on each simplex $T \in \tau$ to find $\varphi_{T} \in$ $\mathrm{C}_{c}^{\infty}\left(T, \mathbb{R}^{N}\right)$ with

$$
f_{T} F\left(\nabla a+\nabla \varphi_{T}\right) \mathrm{d} x<F^{\mathrm{qc}}(\nabla a)+\varepsilon .
$$

Put

$$
\phi=a+\sum_{T \in \tau_{\Omega}} \varphi_{T}
$$

and note that the sum is finite, so $\phi$ is Lipschitz and compactly supported in $\bigcup_{T \in \tau_{\Omega}} \bar{T}$. Furthermore,

$$
\Theta_{q}^{\Omega}(t)+2 \varepsilon>f_{\Omega} F^{\mathrm{qc}}(\nabla a) \mathrm{d} x>f_{\Omega} F(\nabla \phi) \mathrm{d} x-\varepsilon
$$

and the convexity of $z \mapsto|z|^{q}$ yields by Jensen's inequality for each simplex $T \in \tau_{\Omega}$,

$$
f_{T}\left|\nabla a+\nabla \varphi_{T}\right|^{q} \mathrm{~d} x \geq f_{T}|\nabla a|^{q} \mathrm{~d} x,
$$

and therefore

$$
\begin{aligned}
\int_{\Omega}|\nabla \phi|^{q} \mathrm{~d} x & =\sum_{T \in \tau_{\Omega}} \int_{T}\left|\nabla a+\nabla \varphi_{T}\right|^{q} \mathrm{~d} x \\
& \geq \sum_{T \in \tau_{\Omega}} \int_{T}|\nabla a|^{q} \mathrm{~d} x \\
& =\int_{\Omega}|\nabla a|^{q} \mathrm{~d} x \\
& \geq t \mathcal{L}^{n}(\Omega) .
\end{aligned}
$$

Finally we realize the distribution of $\nabla \phi$ on $\omega$ by use of Lemma 2.1. The proof is complete. 
We can exploit the resemblance of the definition of $\Theta(t)$ with the Dacorogna formula for the quasiconvex envelope further, and copying the relevant proofs we easily get the following result which is the key to the proof of Proposition 1.1. Note also that the proof actually works in a much more general situation. In particular one could relax the growth condition (1.1) on $F$ and merely require that $F(z) \geq-k\left(|z|^{p}+1\right)$ for all $z$.

Proposition 3.2. Under the assumptions of Definition 3.2 the auxiliary function $\Theta=\Theta_{q}:[0, \infty) \rightarrow \mathbb{R} \cup\{-\infty\}$ is convex.

Proof. We may assume that $F$ is quasiconvex so that $\Theta(t) \geq F(0)>-\infty$ for all $t \geq 0$. Let $t_{0}, t_{1} \geq 0$ and $\lambda \in(0,1)$. Denote $Q=(0,1)^{n}$ and let $\varepsilon>0$. Take $\varphi_{i} \in \overline{\mathrm{W}}_{0}^{1, p}\left(Q, \mathbb{R}^{N}\right)$ such that

$$
\int_{Q}\left|\nabla \varphi_{i}\right|^{q} \mathrm{~d} x \geq t_{i} \quad \text { and } \quad \int_{Q} F\left(\nabla \varphi_{i}\right) \mathrm{d} x<\Theta\left(t_{i}\right)+\varepsilon .
$$

Put $Q_{0}=(\lambda, 1) \times(0,1)^{n-1}, Q_{1}=(0, \lambda) \times(0,1)^{n-1}$ and write $Q_{i}=\bigcup_{j=1}^{\infty}\left(x_{i, j}+\right.$ $\left.r_{i, j} \bar{Q}\right)$ (disjoint union). If we let

$$
\phi_{i}(x)=\sum_{j=1}^{\infty} r_{i, j} \varphi_{i}\left(\frac{x-x_{i, j}}{r_{i, j}}\right), \quad x \in Q_{i},
$$

then $\phi_{i} \in \mathrm{W}_{0}^{1, p}\left(Q_{i}, \mathbb{R}^{N}\right)$ (for instance by checking absolute convergence of the series),

$$
f_{Q_{i}}\left|\nabla \phi_{i}\right|^{q} \mathrm{~d} x \geq t_{i} \quad \text { and } \quad f_{Q_{i}} F\left(\nabla \phi_{i}\right) \mathrm{d} x<\Theta\left(t_{i}\right)+\varepsilon .
$$

Hence with

$$
\phi:= \begin{cases}\phi_{0} & \text { on } Q_{0} \\ \phi_{1} & \text { on } Q_{1}\end{cases}
$$

we have $\phi \in \mathrm{W}_{0}^{1, p}\left(Q, \mathbb{R}^{N}\right)$ satisfying

$$
\begin{aligned}
\int_{Q}|\nabla \phi|^{q} \mathrm{~d} x & =\int_{Q_{0}}\left|\nabla \phi_{0}\right|^{q} \mathrm{~d} x+\int_{Q_{1}}\left|\nabla \phi_{1}\right|^{q} \mathrm{~d} x \\
& \geq t_{0}(1-\lambda)+t_{1} \lambda
\end{aligned}
$$

and

$$
\begin{aligned}
\int_{Q} F(\nabla \phi) \mathrm{d} x & =\int_{Q_{0}} F\left(\nabla \phi_{0}\right) \mathrm{d} x+\int_{Q_{1}} F\left(\nabla \phi_{1}\right) \mathrm{d} x \\
& <(1-\lambda) \Theta\left(t_{0}\right)+\lambda \Theta\left(t_{1}\right)+\varepsilon
\end{aligned}
$$

Consequently, $\Theta\left((1-\lambda) t_{0}+\lambda t_{1}\right) \leq(1-\lambda) \Theta\left(t_{0}\right)+\lambda \Theta\left(t_{1}\right)$.

The next result shows that coercivity is preserved under mollification. An inspection of the easy proof reveals that in order to establish $\mathrm{L}^{q}$ coercivity one may always assume that the integrand is smooth. 
Lemma 3.2. Let $\Phi \in \mathrm{C}_{c}^{\infty}\left(\mathbb{R}^{N \times n}\right), \Phi \geq 0$ and $\Phi \neq 0$. Then under the assumptions of Definition 3.2 we have that $F$ is $\mathrm{L}^{q}$ coercive if and only if $\Phi * F$ is $\mathrm{L}^{q}$ coercive.

Proof. With $Q=(0,1)^{n}$ and $\varphi \in \mathrm{W}_{0}^{1, p}\left(Q, \mathbb{R}^{N}\right)$ we have by Fubini's theorem

$$
\int_{Q}(\Phi * F)(\nabla \varphi(x)) \mathrm{d} x=\int_{\mathbb{R}^{N \times n}} \int_{Q} F(\nabla \varphi(x)-z) \mathrm{d} x \Phi(z) \mathrm{d} z .
$$

The proof is easily completed using (3.1), (3.2) and that the support of $\Phi$ is bounded. We leave the details to the interested reader.

With the above observations in place we finally turn to the main task of this section.

Proof of Proposition 1.1. First note that the implications (i) $\Rightarrow$ (ii), (iii) $\Rightarrow$ (iv), (iii) $\Rightarrow$ (i), (iv) $\Rightarrow$ (ii) are all obvious. That (ii) $\Rightarrow$ (i) is the content of Proposition 3.1. The proof of (iv) $\Rightarrow$ (iii) is similar, but simpler, and so is omitted here. There are 3 implications left to prove.

(i) $\Rightarrow$ (iv): Assume $F$ is $\mathrm{L}^{q}$ coercive and consider the auxiliary function $\Theta(t)$ from Definition 3.2. The coercivity means that $\Theta(t) \rightarrow \infty$ as $t \rightarrow \infty$, and since according to Proposition 3.2 the function $\Theta$ is convex we can find $c_{1}>0, c_{2} \in \mathbb{R}$ so $\Theta(t) \geq c_{1} t+c_{2}$ for all $t \geq 0$. If $\varphi \in \mathrm{W}_{0}^{1, p}\left(\Omega, \mathbb{R}^{N}\right)$ we may take $t=\|\nabla \varphi\|_{q, \Omega}^{q}$ to conclude that $F$ is $\mathrm{L}^{q}$ mean coercive.

(v) $\Rightarrow$ (iii): If $F-\delta|\cdot|^{q}$ is quasiconvex at $z_{0} \in \mathbb{R}^{N \times n}$, then

$$
F\left(z_{0}\right)-\delta\left|z_{0}\right|^{q} \leq f_{\Omega}\left(F\left(z_{0}+\nabla \varphi(x)\right)-\left|z_{0}+\nabla \varphi(x)\right|^{q}\right) \mathrm{d} x
$$

for all $\varphi \in \mathrm{C}_{c}^{\infty}\left(\Omega, \mathbb{R}^{N}\right)$. This is the $\mathrm{L}^{q}$ mean coercivity condition (1.5) on the Dirichlet class $\mathrm{W}_{g}^{1, p}\left(\Omega, \mathbb{R}^{N}\right)$ corresponding to $g(x)=z_{0} x, c_{1}=\delta$ and $c_{2}=$ $f\left(z_{0}\right)-\delta\left|z_{0}\right|^{q}$.

(iii) $\Rightarrow(\mathrm{v})$ : We assume that (1.5) holds with $g=0$. Take any $\delta \in\left(0, c_{1}\right)$ and put $G(z)=F(z)-\delta|z|^{q}$ for $z \in \mathbb{R}^{N \times n}$. From Lemma 3.1 we infer that

$$
f_{\Omega} G(\nabla \varphi) \mathrm{d} x \geq f_{\Omega} G^{\mathrm{qc}}(\nabla \varphi) \mathrm{d} x \geq\left(c_{1}-\delta\right) f_{\Omega}|\nabla \varphi|^{q} \mathrm{~d} x+c_{2}
$$

for all $\varphi \in \mathrm{W}_{0}^{1, p}\left(\Omega, \mathbb{R}^{N}\right)$. Take a minimizing sequence $\left(\varphi_{j}\right) \subset \mathrm{C}_{c}^{\infty}\left(\Omega, \mathbb{R}^{N}\right)$ for $G^{\mathrm{qc}}(0)$ :

$$
G^{\mathrm{qc}}(0) \leq f_{\Omega} G^{\mathrm{qc}}\left(\nabla \varphi_{j}\right) \mathrm{d} x \leq f_{\Omega} G\left(\nabla \varphi_{j}\right) \mathrm{d} x \searrow G^{\mathrm{qc}}(0) .
$$

The probability distributions

$$
\nu_{j}=\left(\nabla \varphi_{j}\right)_{\#}\left(\frac{\mathcal{L}^{n}\lfloor\Omega}{\mathcal{L}^{n}(\Omega)}\right)
$$

all have centre of mass at 0 and uniformly bounded $q$-th moments. Passing to a subsequence if necessary we can assume that $\nu_{j} \rightarrow \nu$ weakly* in $\mathrm{C}_{0}\left(\mathbb{R}^{N \times n}\right)^{*}$, 
where $\nu$ is a probability measure on $\mathbb{R}^{N \times n}$ with a finite $q$-th moment (and centre of mass at 0 provided $q>1)$. Note that $H(z)=G(z)-G^{\mathrm{qc}}(z)$ is continuous and nonnegative, so by standard results we get

$$
0 \leq \int_{\mathbb{R}^{N \times n}} H \mathrm{~d} \nu \leq \liminf _{j \rightarrow \infty} \int_{\mathbb{R}^{N \times n}} H \mathrm{~d} \nu_{j}=0 .
$$

But then $G=G^{\mathrm{qc}}$ on the support $\operatorname{spt}(\nu)$, that is, $F-\delta|\cdot|^{q}$ is quasiconvex at each $z_{0} \in \operatorname{spt}(\nu)$. For $q>1$, where $\nu$ has centre of mass at 0 , it then follows in a routine manner that 0 belongs to the closed convex hull of $\operatorname{spt}(\nu)$. In fact, since we could have started at any $z \in \mathbb{R}^{N \times n}$ instead of at 0 , we infer that in the case $q>1$ the integrand $F-\delta|\cdot|^{q}$ is quasiconvex at each point $z_{0}$ of a set whose closed convex hull contains $z$, and therefore that the set where $F-\delta|\cdot|{ }^{q}$ is quasiconvex has $\mathbb{R}^{N \times n}$ as its closed convex hull. (In the obvious terminology the set where $F-\delta|\cdot|^{q}$ is quasiconvex has $\mathbb{R}^{N \times n}$ as its $\mathrm{L}^{q}$ quasiconvex hull when $q>1$.)

\section{Examples of $\mathrm{L}^{p}$ coercive integrands}

In this section we investigate coercivity in the context of nonnegative positively $p$-homogeneous integrands $F$. In this case the $\mathrm{L}^{p}$ coercivity, as noted in $[32,38]$, only depends on the zero set $\{F=0\}$ and not on other features of $F$ :

Lemma 4.1. Let $F, G: \mathbb{R}^{N \times n} \rightarrow \mathbb{R}$ be nonnegative and positively $p$-homogeneous continuous integrands for an exponent $p \geq 1$. Then we have $\{F=0\} \subseteq\{G=0\}$ if and only if

$$
\forall \varepsilon>0 \exists c<\infty \text { such that } G(z) \leq \varepsilon|z|^{p}+c F(z) \quad \forall z \in \mathbb{R}^{N \times n} .
$$

We leave the easy verification of the lemma to the reader. For a nonnegative positively $p$-homogeneous integrand $F$ we let $Z_{F}=\left\{z \in \mathbb{R}^{N \times n}: F(z)=0\right\}$ and note that $Z_{F}$ is a closed cone in $\mathbb{R}^{N \times n}$. Now by Lemma $4.1, F$ is $\mathrm{L}^{p}$ coercive if and only if the $p$-th power of the euclidean distance function to the zero set $Z_{F}, z \mapsto \operatorname{dist}^{p}\left(z, Z_{F}\right)$, is $\mathrm{L}^{p}$ coercive. Here we recall that the $p$-th power distance function is quasiconvex (in fact, is rank one convex) if and only if $Z_{F}$ is a convex set (see [41] and [9]). Hence to get the geometric flavour of the $p$-th power distance function we must necessarily, when $Z_{F}$ is nonconvex, work with non-rank one convex integrands. Let us also recall that when $F$ is a nonnegative, positively $p$-homogeneous and convex integrand, then $z \mapsto F(z)^{\frac{1}{p}}$ is convex too: it is the Minkowski functional for the set $\left\{z \in \mathbb{R}^{N \times n}: F(z) \leq 1\right\}$. Such a result is false for general quasiconvex nonnegative positively $p$-homogeneous integrands $F$ where $z \mapsto F(z)^{\alpha}$ need not be rank one convex for any exponent $\alpha \in(0,1)$. Indeed, that integrands $F$ for which $F^{\frac{1}{p}}$ is $\mathrm{L}^{1}$ coercive are special can be seen from the following result.

Lemma 4.2. Let $G$ be a nonnegative positively 1-homogeneous integrand. Then $G$ is $\mathrm{L}^{1}$ coercive if and only if $G$ is demicoercive: there exist $z_{0} \in \mathbb{R}^{N \times n}$ and 
$\varepsilon>0$ such that $G(z) \geq z_{0} \cdot z+\varepsilon|z|$ for all $z \in \mathbb{R}^{N \times n}$. If we strengthen the assumptions on $G$ and require that it is 1-homogeneous (so $G(t z)=|t| G(z)$ for all $t \in \mathbb{R})$, then $G$ is $\mathrm{L}^{1}$ coercive if and only if it is 1-coercive: there exists $\varepsilon>0$ such that $G(z) \geq \varepsilon|z|$ for all $z \in \mathbb{R}^{N \times n}$.

This is a special case of [21, Theorems 1.1 and 5.1], and so we omit the proof here.

As in [38] we adopt the following terminology:

Definition 4.1. A closed cone $\mathcal{K}$ in $\mathbb{R}^{N \times n}$ is called $\mathrm{L}^{p}$ coercive if $z \mapsto \operatorname{dist}^{p}(z, \mathcal{K})$ is $\mathrm{L}^{p}$ coercive.

It is shown in [38, Theorem 2.3] that the set of exponents $p \geq 1$ for which a given closed cone $\mathcal{K}$ is $\mathrm{L}^{p}$ coercive is an open subset of $[1, \infty)$. The proof makes use of the celebrated stability result of [19]. The situation for $\mathrm{L}^{q}, q<p$, and $\mathrm{L}^{p, \infty}$ coercivity seems more complicated and is bound to depend also on other features of the positively $p$-homogeneous integrand $F$, than merely its zero set.

Note that Proposition 2.1 yields that a necessary condition for a closed cone to be $\mathrm{L}^{p}$ coercive for an exponent $p>1$ is that it contains no rank one matrices. This condition will in general not suffice and there are many interesting open questions to address in this regard, but here we shall confine ourselves to give a few elementary examples in the situation where the cone is in addition assumed convex.

Proposition 4.1. Let $\mathcal{K}$ be a closed convex cone in $\mathbb{R}^{N \times n}$. Then $\mathcal{K}$ is $\mathrm{L}^{2}$ coercive if and only if $\mathcal{K}$ contains no rank one matrices.

Proof. We have already remarked on the only if direction, so consider a closed convex cone $\mathcal{K}$ with $\operatorname{rank}(z) \neq 1$ for all $z \in \mathcal{K}$. Put

$$
\alpha=\inf \left\{\operatorname{dist}^{2}(z, \mathcal{K}):|z|=1=\operatorname{rank}(z)\right\} .
$$

Clearly, $\operatorname{dist}^{2}(z, \mathcal{K}) \geq \alpha|z|^{2}$ for all $z$ with $\operatorname{rank}(z) \leq 1$. Since $z \mapsto \operatorname{rank}(z)$ is lower semicontinuous the set $\{z:|z|=1=\operatorname{rank}(z)\}$ is easily seen to be compact, hence the infimum defining $\alpha$ is attained and therefore $\alpha>0$. Put $Y=(0,1)^{n}$ and let $\varphi$ be an $\mathbb{R}^{N}$-valued trigonometric polynomial on $Y: \varphi(x)=$ $\sum_{j \in J} c_{j} e^{2 \pi \mathrm{i} j \cdot x}$, where $J \subset \mathbb{Z}^{n}$ is a finite non-empty set and $c_{j}=c_{j}^{\prime}+\mathrm{i} c_{j}^{\prime \prime} \in \mathbb{C}^{N}$. If $F(z)=\operatorname{dist}(z, \mathcal{K})$, then $F$ is convex and positively 1-homogeneous and so admits the representation $F(z)=\sup _{z^{*} \in \partial F(0)} z^{*} \cdot z$, where $\partial F(0)$ is the subdifferential of $F$ at 0 . Consequently, to $\varepsilon \in(0, \alpha)$ we may find $z_{1}^{*}, \ldots, z_{m}^{*} \in \partial F(0), m=$ $m_{\varepsilon} \in \mathbb{N}$ such that

$$
\operatorname{dist}^{2}(z, \mathcal{K}) \leq \varepsilon|z|^{2}+\sup _{1 \leq k \leq m}\left(z_{k}^{*} \cdot z\right)^{2}
$$

holds for all $z \in \mathbb{R}^{N \times n}$. Put

$$
A_{k}=\left\{z \in \mathbb{R}^{N \times n}: \operatorname{dist}^{2}(z, \mathcal{K}) \leq \varepsilon|z|^{2}+\left(z_{k}^{*} \cdot z\right)^{2}\right\}
$$


and note that $\mathbb{R}^{N \times n}=\bigcup_{k=1}^{m} A_{k}$. Now $\nabla \varphi(x)=\sum_{j \in J} c_{j} \otimes(2 \pi \mathrm{i} j) e^{2 \pi \mathrm{i} j \cdot x}$ and so we may estimate

$$
\begin{aligned}
\int_{Y} \operatorname{dist}^{2}(\nabla \varphi, \mathcal{K}) \mathrm{d} x & \geq \sup _{1 \leq k \leq m} \int_{Y}\left(z_{k}^{*} \cdot \nabla \varphi\right)^{2} \mathrm{~d} x \\
& \stackrel{\text { Parseval }}{=} \sup _{1 \leq k \leq m} \sum_{j \in J}\left|z_{k}^{*} \cdot c_{j} \otimes(2 \pi \mathrm{i} j)\right|^{2} \\
& \frac{1}{m} \sum_{k=1}^{m} \sum_{j \in J}\left|z_{k}^{*} \cdot c_{j} \otimes(2 \pi \mathrm{i} j)\right|^{2} \\
& =\frac{1}{m} \sum_{j \in J} \sum_{k=1}^{m}\left(\left|z_{k}^{*} \cdot c_{j}^{\prime} \otimes(2 \pi j)\right|^{2}+\left|z_{k}^{*} \cdot c_{j}^{\prime \prime} \otimes(2 \pi j)\right|^{2}\right) \\
& \geq \frac{\alpha-\varepsilon}{m} \sum_{j \in J}\left(\left|c_{j}^{\prime} \otimes(2 \pi j)\right|^{2}+\left|c_{j}^{\prime \prime} \otimes(2 \pi j)\right|^{2}\right) \\
& \stackrel{\alpha-\varepsilon}{m} \int_{Y}|\nabla \varphi|^{2} \mathrm{~d} x,
\end{aligned}
$$

as required.

Remark 4.1. One may use the above proof to establish the following variant. Let $\mathcal{K}$ be a closed convex cone and fix $z_{0} \in K$. Then there exists a constant $\beta>0$ such that

$$
\int_{Y} \operatorname{dist}^{2}\left(\nabla \varphi+z_{0}, \mathcal{K}\right) \mathrm{d} x \geq \beta \int_{Y}|\nabla \varphi|^{2} \mathrm{~d} x,
$$

holds for all $\varphi \in \mathrm{W}_{0}^{1,2}\left(Y, \mathbb{R}^{N}\right)$ if and only if $\operatorname{rank}\left(z-z_{0}\right) \neq 1$ for all $z \in \mathcal{K}$.

Note that the convexity of $\mathcal{K}$ means that if the above condition holds for a $z_{0} \in \mathcal{K} \backslash\{0\}$, then $\mathcal{K}$ does not contain any rank one matrices.

We elaborate on the remark and strengthen the assumption on the convex cone and require that the condition in Remark 4.1 holds at all matrices $z_{0}$ of the cone:

Theorem 4.1. Let $\mathcal{K}$ be a closed convex cone in $\mathbb{R}^{N \times n}$. If $\mathcal{K}$ contains no rank one connections, that is, if for all $z_{0}, z_{1} \in \mathcal{K}$ we have $\operatorname{rank}\left(z_{1}-z_{0}\right) \neq 1$, then $\mathcal{K}$ is $\mathrm{L}^{p}$ coercive for all $p \in(1, \infty)$.

While we are not aware of any proof for the above result in the literature, the result is hardly new. The second author was told by Kewei Zhang that such a result holds and can be proved using a Fourier multiplier theorem a while ago [42]. Our proof here avoids multipliers but is of course related with such a proof and we are grateful to Kewei Zhang for comments and discussions.

Proof. We start with the observation that a closed convex cone $\mathcal{K}$ contains no rank one connections precisely when the linear subspace $L=\operatorname{span}(\mathcal{K})$ contains 
no rank one matrices. Now obviously we have $\operatorname{dist}^{p}(z, \mathcal{K}) \geq \operatorname{dist}^{p}(z, L)$ for all $z \in \mathbb{R}^{N \times n}$, so the sought $\mathrm{L}^{p}$ coercivity follows trivially if we prove that $\operatorname{dist}^{p}(z, L)$ is $\mathrm{L}^{p}$ coercive. This is what we will do and from now on we assume that $\mathcal{K}$ is a linear subspace of $\mathbb{R}^{N \times n}$ that contains no rank one matrices.

Let $\pi$ denote orthogonal projection of $\mathbb{R}^{N \times n}$ onto $\mathcal{K}^{\perp}$, the orthogonal complement of $\mathcal{K}$ in $\mathbb{R}^{N \times n}$. Then we have $F(z)=|\pi(z)|^{p}$, and for proving the $\mathrm{L}^{p}$ coercivity we shall consider the operator $P: \mathrm{W}_{0}^{1, p}\left(Y, \mathbb{R}^{N}\right) \rightarrow \mathrm{L}^{p}\left(Y, \mathbb{R}^{N}\right)$ given by $P(\varphi)=\pi(\nabla \varphi)$, where we denoted $Y=(0,1)^{n}$.

The assumption that $\mathcal{K}$ contains no rank one matrices now translates to injectivity of $P: \operatorname{ker} P=\{0\}$. To see this we first extend the linear transformation $\pi$ to a linear mapping of $\mathbb{C}^{N \times n}$ in a standard manner: $\pi\left(z^{\prime}+\mathrm{i} z^{\prime \prime}\right):=\pi\left(z^{\prime}\right)+\mathrm{i} \pi\left(z^{\prime \prime}\right)$. Now if for some $\varphi \in \mathrm{W}_{0}^{1, p}$ we have $P(\varphi)=0$, then we compare Fourier coefficients: for $j \in \mathbb{Z}^{n}$ let

$$
c_{j}=c_{j}^{\prime}+\mathrm{i} c_{j}^{\prime \prime}=\int_{Y} \varphi(x) \mathrm{e}^{-2 \pi \mathrm{i} j \cdot x} \mathrm{~d} x .
$$

Then

$$
\int_{Y} \nabla \varphi(x) \mathrm{e}^{-2 \pi \mathrm{i} j \cdot x} \mathrm{~d} x=c_{j} \otimes(2 \pi \mathrm{i} j),
$$

and so by linearity of $\pi$,

$$
\begin{aligned}
0 & =\int_{Y} \pi(\nabla \varphi(x)) \mathrm{e}^{-2 \pi \mathrm{i} j \cdot x} \mathrm{~d} x \\
& =\pi\left(c_{j} \otimes(2 \pi \mathrm{i} j)\right) \\
& =\pi\left(c_{j}^{\prime \prime} \otimes 2 \pi j\right)+\mathrm{i} \pi\left(c_{j}^{\prime} \otimes(-2 \pi j)\right) .
\end{aligned}
$$

But the kernel of $\pi$ contains no rank one matrices, so necessarily $c_{j}=0$ for $j \neq 0$, and we infer that $\varphi=c_{0}$ by uniqueness of Fourier coefficients (see for instance [36, Theorem 1.7(ii)]). Because $\varphi=0$ on $\partial Y$ also $c_{0}=0$ and we have shown that $P$ is injective.

By the Open Mapping theorem the $\mathrm{L}^{p}$ coercivity follows if we show that $P$ has a closed range. In turn this follows by the Closed Range theorem if we show that the dual operator $P^{*}: \mathrm{L}^{p^{\prime}} \rightarrow \mathrm{W}^{-1, p^{\prime}}$ has a closed range. To that end, let $V_{j} \in \mathrm{L}^{p^{\prime}}$ and assume $P^{*}\left(V_{j}\right) \rightarrow T$ in $\mathrm{W}^{-1, p^{\prime}}$. Thus uniformly in $\varphi \in \mathrm{W}_{0}^{1, p}$ with $\|\varphi\|_{\mathrm{W}^{1, p}} \leq 1$ :

$$
\begin{aligned}
\langle T, \varphi\rangle & =\lim _{j \rightarrow \infty}\left\langle V_{j}, \pi(\nabla \varphi)\right\rangle \\
& =\lim _{j \rightarrow \infty}\left\langle\pi\left(V_{j}\right), \nabla \varphi\right\rangle
\end{aligned}
$$

Here $\pi\left(V_{j}\right) \in \mathrm{L}^{p^{\prime}}$ and so by Helmholtz decomposition in $\mathrm{L}^{p^{\prime}}$,

$$
\pi\left(V_{j}\right)=\nabla \phi_{j}+\sigma_{j}
$$

where $\phi_{j} \in \mathrm{W}_{0}^{1, p^{\prime}}$ and $\sigma_{j} \in \mathrm{L}^{p^{\prime}}, \operatorname{div} \sigma_{j}=0$ (row-wise and in distributional sense). 
Integrating by parts this allows us to write $P^{*}\left(V_{j}\right)=P^{*}\left(\nabla \phi_{j}\right)$ and

$$
\begin{aligned}
\langle T, \varphi\rangle & =\lim _{j \rightarrow \infty}\left\langle P^{*}\left(\nabla \phi_{j}\right), \varphi\right\rangle \\
& =\lim _{j \rightarrow \infty} \int_{Y} \nabla \phi_{j} \cdot \nabla \varphi \mathrm{d} x \\
& =\lim _{j \rightarrow \infty} \int_{Y} \nabla \phi_{j} \cdot w \mathrm{~d} x,
\end{aligned}
$$

where $w \in \mathrm{L}^{p}$ is any field whose $\mathrm{L}^{p}$ Helmholtz projection onto gradient fields is $\nabla \varphi$. If $c_{p}$ denotes the operator norm of this Helmholtz projection onto gradients in $\mathrm{L}^{p}$, then we may take

$$
w=w_{j}=\frac{\left|\nabla \phi_{j}\right|^{p^{\prime}-2} \nabla \phi_{j}}{c_{p}\left\|\nabla \phi_{j}\right\|_{p^{\prime}}^{\frac{p^{\prime}}{p}}}
$$

above to conclude that $\nabla \phi_{j}$ is bounded in $\mathrm{L}^{p^{\prime}}$ and therefore, by reflexivity, up to a subsequence, is weakly convergent. This completes the proof.

\section{$5 \quad$ Semicontinuity for signed integrands}

Theorem 5.1. Let $F: \mathbb{R}^{N \times n} \rightarrow \mathbb{R}$ be a quasiconvex integrand satisfying the growth condition (1.1). Fix exponents $1 \leq q \leq p$ such that

$$
\left(1-\frac{1}{n}\right) p<q .
$$

If $u, u_{j} \in \mathrm{W}_{g}^{1, p}\left(\Omega, \mathbb{R}^{N}\right), u_{j} \rightarrow u$ in $\mathrm{L}^{1}$ and $\sup _{j}\left\|\nabla u_{j}\right\|_{\mathrm{L}^{q}}<\infty$, then

$$
\liminf _{j \rightarrow \infty} \int_{\Omega} F\left(\nabla u_{j}\right) \mathrm{d} x \geq \int_{\Omega} F(\nabla u) \mathrm{d} x .
$$

The result is well-known for integrands $F$ satisfying additionally that

$$
\liminf _{|z| \rightarrow \infty} \frac{F(z)}{|z|^{q}} \geq 0 .
$$

In view of the many examples of signed and positively $p$-homogeneous quasiconvex integrands this does not exhaust all possibilities and so our result here closes that gap. We do not contribute to the interesting issue of what the general optimal bounds for the exponent $q$ might be. The reader will notice that this must be so when seeing the proof. It relies on several standard facts and is merely a combination of well-known proofs for lower semicontinuity of quasiconvex integrals going back to Morrey [30], Meyers [28], Fusco [14], Fonseca \& Malý [12] (see also [23, 34] for $q=1<p<\frac{n}{n-1}$ and [14] for $p=q>1$ and [25] for $p=q=1$ ). We start with the following consequence of Fubini's theorem. 
Lemma 5.1. Let $\sigma^{n}=\operatorname{co}\left\{0, e_{1}, \ldots, e_{n}\right\}$ denote the standard simplex in $\mathbb{R}^{n}$ and let $e \in \mathbb{S}^{n-1}$ be a unit vector that is not tangent to any of the $n+1$ faces of $\sigma^{n}$, that is, $e \cdot e_{j} \neq 0$ for all $0 \leq j \leq n$, where $e_{0}:=\left(e_{1}+\ldots+e_{n}\right) / \sqrt{n}$. For a nonnegative locally integrable function $f: \mathbb{R}^{n} \rightarrow[0, \infty)$ and $r>0$ we have that

$$
\int_{0}^{r} \int_{t e+\partial \sigma^{n}} f(x) \mathrm{d} \mathcal{H}^{n-1} \mathrm{~d} t \leq \frac{n+1}{\min _{0 \leq j \leq n}\left|e \cdot e_{j}\right|} \int_{B_{r}\left(\sigma^{n}\right)} f(x) \mathrm{d} x .
$$

Proof. Let $\mathcal{F}_{j}$ denote the face of $\sigma^{n}$ opposite the vertex $e_{j}$ for $j \neq 0$, and $\mathcal{F}_{0}$ the face opposite the vertex 0 . Then clearly

$$
\int_{0}^{r} \int_{t e+\partial \sigma^{n}} f(x) \mathrm{d} \mathcal{H}^{n-1} \mathrm{~d} t=\sum_{j=0}^{n} \int_{0}^{r} \int_{t e+\mathcal{F}_{j}} f(x) \mathrm{d} \mathcal{H}^{n-1} \mathrm{~d} t .
$$

Write $e=\left(e \cdot e_{j}\right) e_{j}+e_{j}^{\perp}$ in the obvious notation, and use Fubini's theorem to estimate

$$
\int_{0}^{r} \int_{t e+\mathcal{F}_{j}} f(x) \mathrm{d} \mathcal{H}^{n-1} \mathrm{~d} t \leq \frac{1}{\left|e \cdot e_{j}\right|} \int_{B_{r}\left(\mathcal{F}_{j}\right)} f(x) \mathrm{d} x .
$$

The conclusion follows immediately from this.

Lemma 5.2. Let $\sigma^{n}=\operatorname{co}\left\{0, e_{1}, \ldots, e_{n}\right\}$ denote the standard simplex in $\mathbb{R}^{n}$. Then there exists a linear extension operator $E: \mathrm{W}^{1, p}\left(\partial \sigma^{n}, \mathbb{R}^{N}\right) \rightarrow \mathrm{W}_{\text {loc }}^{1, p}\left(\mathbb{R}^{n}, \mathbb{R}^{N}\right)$ with the properties: $E\left(\left.a\right|_{\partial \sigma^{n}}\right)=$ a whenever $a: \mathbb{R}^{n} \rightarrow \mathbb{R}^{N}$ is affine, and for each $r>0$,

$$
\int_{B_{r}(0)}\left|\nabla E\left(v_{j}\right)\right|^{p} \mathrm{~d} x \rightarrow 0
$$

whenever $v_{j} \rightarrow 0$ in $\mathrm{W}^{1, q}\left(\partial \sigma^{n}, \mathbb{R}^{N}\right)$. Recall that $\left(1-\frac{1}{n}\right) p<q$. We also have a bound:

$$
\int_{B_{10}\left(\sigma^{n}\right)}|\nabla E(v)|^{p} \mathrm{~d} x \leq c\left(\int_{\partial \sigma^{n}}\left|\nabla_{\tau} v\right|^{q} \mathrm{~d} \mathcal{H}^{n-1}\right)^{\frac{p}{q}}
$$

for all $v \in \mathrm{W}^{1, p}\left(\partial \sigma^{n}, \mathbb{R}^{N}\right)$, where $c=c(n, p, q)$. Here $\nabla_{\tau} v$ denotes the tangential weak derivative of $v$ on the faces of $\partial \sigma^{n}$.

The operator $E$ can be constructed by local mollification and a partition of unity that is subordinate to a suitable Whitney covering of the complement $\mathbb{R}^{n} \backslash \partial \sigma^{n}$. We omit the details here, and instead refer the reader to [12] for a more sophisticated construction and to [22] for related results.

Proof of Theorem 5.1. In view of the growth condition (1.1) we may extract a subsequence, relabel the subsequence and then assume that

$$
\liminf _{j \rightarrow \infty} \int_{\Omega} F\left(\nabla u_{j}\right) \mathrm{d} x=\lim _{j \rightarrow \infty} \int_{\Omega} F\left(\nabla u_{j}\right) \mathrm{d} x=\ell \in \mathbb{R} .
$$

Let $\varepsilon \in(0,1)$. We may consider $u, u_{j} \in \mathrm{W}^{1, p}\left(\mathbb{R}^{n}, \mathbb{R}^{N}\right)$ simply by taking $u=$ $g$ and $u_{j}=g$ outside $\Omega$. Then using a standard smooth mollifier we find 
$\tilde{u} \in\left(\mathrm{C}^{\infty} \cap \mathrm{W}^{1, p}\right)\left(\mathbb{R}^{n}, \mathbb{R}^{N}\right)$ with $\|u-\tilde{u}\|_{\mathrm{W}^{1, p}}<\varepsilon / 2$. By the smoothness of $\tilde{u}$ we find a $\delta \in(0,1)$ such that for each regular and uniform triangulation $\tau$ of $\mathbb{R}^{n}$ the piecewise affine Lipschitz mapping $a=a_{\tau}: \mathbb{R}^{n} \rightarrow \mathbb{R}^{N}$ obtained by Lagrange interpolating $\tilde{u}$ on the nodes of $\tau$ satisfies $\|\tilde{u}-a\|_{\mathrm{W}^{1, p}\left(B_{2}(\Omega)\right)}<\varepsilon / 2$ provided all the simplices $T \in \tau$ satisfy $\operatorname{diam} T<\delta$. Fix such a regular and uniform triangulation $\tau$ of $\mathbb{R}^{n}$. Because of the regularity and uniformity of $\tau$ each simplex $T \in \tau$ is congruent to a finite number of simplices and so there exists a unit vector $e \in \mathbb{S}^{n-1}$ which isn't tangent to any of the faces of any simplex $T \in \tau$. Consequently, by an affine change of variables in $\mathbb{R}^{n}$ we can employ Lemma 5.1 whereby we find constants $c_{1}>0$ and $c_{2} \geq 1$, depending only on $n, e, \tau$, such that with $\tau_{\Omega}=\left\{T \in \tau: T \cap B_{1}(\Omega) \neq \emptyset\right\}$ we have

$$
\begin{aligned}
\sum_{T \in \tau_{\Omega}} \int_{0}^{\delta} \liminf _{j \rightarrow \infty} \int_{t e+\partial T}\left|\nabla u_{j}\right|^{q} \mathrm{~d} \mathcal{H}^{n-1} \mathrm{~d} t & \stackrel{\text { Fatou }}{\leq} \liminf _{j \rightarrow \infty} \sum_{T \in \tau_{\Omega}} \int_{0}^{\delta} \int_{t e+\partial T}\left|\nabla u_{j}\right|^{q} \mathrm{~d} \mathcal{H}^{n-1} \mathrm{~d} t \\
& \leq \sum_{T \in \tau_{\Omega}} c_{1} \int_{B_{c_{2} \delta}(T)}\left|\nabla u_{j}\right|^{q} \mathrm{~d} x \\
& \leq \liminf _{j \rightarrow \infty} \int_{B_{2 c_{2} \delta}(\Omega)}\left|\nabla u_{j}\right|^{q} \mathrm{~d} x<\infty,
\end{aligned}
$$

where the constant $c$ depends on $c_{1}, c_{2}, \delta$ (and it exists because $\tau_{\Omega}$ is finite; in fact, a slightly more refined bound, that we don't need here, would rely on the finite overlap property of the dilated simplices). The outcome of the above string of inequalities is that there exist $t \in(0, \delta)$ and a subsequence of $u_{j}$ (not relabelled) such that $\left.u\right|_{\partial T},\left.u_{j}\right|_{\partial T} \in \mathrm{W}^{1, p}\left(\partial T, \mathbb{R}^{N}\right)$ and $\left.\left.u_{j}\right|_{\partial T} \rightarrow u\right|_{\partial T}$ in $\mathrm{W}^{1, q}\left(\partial T, \mathbb{R}^{N}\right)$ for each simplex $T \in \tau_{\Omega}$. Using an affine change of variables we are in a position to employ Lemma 5.2 on each translated simplex te $+T$, $T \in \tau_{\Omega}$. Of course, $\left\{t e+T: T \in \tau_{\Omega}\right\}$ is a triangulation of a neighbourhood of $\bar{\Omega}$ and henceforth we shall denote it by $\tau$. Likewise, the piecewise affine mapping obtained by Lagrange interpolating $\tilde{u}$ on the nodes of $\tau$ is denoted by $a$, and using the triangle inequality and the assumptions we have

$$
\int_{\bigcup \tau_{\Omega}}\left(|\nabla u-\nabla a|^{p}+|u-a|^{p}\right) \mathrm{d} x<\varepsilon .
$$

Fix a simplex $T \in \tau_{\Omega}$. Let $A: \mathbb{R}^{n} \rightarrow \mathbb{R}^{n}$ be an affine mapping such that $A(T)=$ $\sigma^{n}$. Then $\left.\left(u \circ A^{-1}\right)\right|_{\partial \sigma^{n}},\left.\left(u_{j} \circ A^{-1}\right)\right|_{\partial \sigma^{n}} \in \mathrm{W}^{1, p}\left(\partial \sigma^{n}, \mathbb{R}^{N}\right)$ and so using Lemma 5.2 we have with $w(x)=E\left(\left.u \circ A^{-1}\right|_{\partial \sigma^{n}}\right)(A x), w_{j}(x)=E\left(\left.u_{j} \circ A^{-1}\right|_{\partial \sigma^{n}}\right)(A x)$ that $w=u, w_{j}=u_{j}$ on $\partial T$ and $w_{j} \rightarrow w$ in $\mathrm{W}^{1, p}\left(B_{1}(\Omega), \mathbb{R}^{N}\right)$. Let $\sigma>1$ and denote by $\sigma T$ the simplex obtained by dilating $T$ by $\sigma$ about its centre. Define for a standard cut-off function $\rho$ between $T$ and $\sigma T$,

$$
v_{j}= \begin{cases}u_{j}+b & \text { in } T \\ a_{T}+\rho\left(w_{j}-a_{T}+b_{j}\right) & \text { in } \sigma T \backslash T,\end{cases}
$$

where $a_{T}$ denotes the affine mapping satisfying $a=a_{T}$ on $T$ and $b_{j} \in \mathbb{R}^{N}$ is chosen so the integral $\int_{\sigma T \backslash T}\left(w_{j}-a_{T}+b_{j}\right) \mathrm{d} x=0$. Then $v_{j}-a_{T} \in \mathrm{W}_{0}^{1, p}\left(\sigma T, \mathbb{R}^{N}\right)$ 
and so quasiconvexity at $\nabla a_{T}$ and the growth condition (1.1) yield

$$
\begin{aligned}
F\left(\nabla a_{T}\right) \mathcal{L}^{n}(\sigma T) & \leq \int_{\sigma T} F\left(\nabla v_{j}\right) \mathrm{d} x \\
& =\int_{T} F\left(\nabla u_{j}\right) \mathrm{d} x+\int_{\sigma T \backslash T} F\left(\nabla\left(a_{T}+\rho\left(w_{j}-a_{T}+b_{j}\right)\right)\right) \mathrm{d} x \\
& =I_{j}+I I_{j} .
\end{aligned}
$$

Here the second term can be estimated using (1.1) and the assumptions:

$$
\begin{aligned}
& \left|I I_{j}\right| \leq \quad \leq \int_{\sigma T \backslash T}\left(1+\left|\nabla a_{T}\right|^{p}+\left|\nabla\left(w_{j}-a_{T}\right)\right|^{p}+\left|w_{j}-a_{T}+b_{j}\right|^{p}|\nabla \rho|^{p}\right) \mathrm{d} x \\
& \stackrel{\text { Poincaré }}{\leq} \quad c\left(\sigma^{n}-1\right)\left(1+\left|\nabla a_{T}\right|^{p}\right) \mathcal{L}^{n}(T)+c \int_{\sigma T \backslash T}\left|\nabla w_{j}-\nabla a_{T}\right|^{p} \mathrm{~d} x \\
& \stackrel{\text { Lemma 5.2 }}{\rightarrow} c\left(\sigma^{n}-1\right)\left(1+\left|\nabla a_{T}\right|^{p}\right) \mathcal{L}^{n}(T)+c \int_{\sigma T \backslash T}\left|\nabla w-\nabla a_{T}\right|^{p} \mathrm{~d} x \\
& \stackrel{\text { Lemma 5.2 }}{\leq} c\left(\sigma^{n}-1\right)\left(1+\left|\nabla a_{T}\right|^{p}\right) \mathcal{L}^{n}(T)+c \int_{T}|\nabla u-\nabla a|^{p} \mathrm{~d} x .
\end{aligned}
$$

It follows that the sequence $I_{j}$ is bounded, and so summing over the (finite number of) simplices $T \in \tau_{\Omega}$ we find

$$
\begin{aligned}
\sigma^{n} \int_{\bigcup \tau_{\Omega}} F(\nabla a) \mathrm{d} x \leq & \liminf _{j \rightarrow \infty} \int_{\bigcup \tau_{\Omega}} F\left(\nabla u_{j}\right) \mathrm{d} x \\
& +c\left(\sigma^{n}-1\right) \int_{\bigcup \tau_{\Omega}}\left(1+|\nabla a|^{p}\right) \mathrm{d} x \\
& +c \int_{\bigcup \tau_{\Omega}}|\nabla u-\nabla a|^{p} \mathrm{~d} x \\
\leq & \liminf _{j \rightarrow \infty} \int_{\Omega} F\left(\nabla u_{j}\right) \mathrm{d} x+\int_{\bigcup \tau_{\Omega} \backslash \Omega} F(\nabla g) \mathrm{d} x \\
& +c \varepsilon+c\left(\sigma^{n}-1\right) \int_{\bigcup \tau_{\Omega}}\left(1+|\nabla u|^{p}\right) \mathrm{d} x .
\end{aligned}
$$

Finally, using that $u=g$ off $\Omega$, the way $a$ was chosen and the growth condition (1.1) we estimate

$$
\begin{aligned}
\sigma^{n} \int_{\Omega} F(\nabla u) \mathrm{d} x \leq & \liminf _{j \rightarrow \infty} \int_{\Omega} F\left(\nabla u_{j}\right) \mathrm{d} x \\
& +c \varepsilon+c\left(\sigma^{n}-1\right) \int_{B_{2}(\Omega)}\left(1+|\nabla u|^{p}\right) \mathrm{d} x
\end{aligned}
$$

and the conclusion follows.

Remark 5.1. Let us note that the usual blow-up method [13] would require some modification if one wanted to use it here in the signed case. Also note 
that in the non-signed case (5.2) the blow-up method automatically yields lower semicontinuity when the limit mapping $u$ is in $\mathrm{W}^{1, q}$ whereas we here require $u$ to be in $\mathrm{W}^{1, p}$. We have not attempted to extend the variational integral by lower semicontinuity as in [26] to $\mathrm{W}^{1, q}$ or $\mathrm{BV}$ in case $q=1$, as the applications given here do not seem to require this. But it is easy to imagine scenarios where this would be necessary, besides it being intrinsically interesting of course.

Finally, let us emphasize that in the signed case (and even for $p=q>1$ ) the restriction to Dirichlet classes $\mathrm{W}_{g}^{1, p}$ is essential: the lower semicontinuity result of Proposition 5.1 fails for sequences $u_{j}$ merely in $\mathrm{W}^{1, p}$ (refer again to the example [8, Remark 8.5(iii)]).

\section{References}

[1] E. Acerbi and N. Fusco, Semicontinuity problems in the calculus of variations, Arch. Ration. Mech. Anal. 86 (1984), no. 2, 125145 .

[2] L. Ambrosio, N. Fusco and D. Pallara, Functions of bounded variation and free discontinuity problems, Oxford Mathematical Monographs, The Clarendon Press, Oxford University Press, New York, 2000.

[3] G. Anzellotti, G. Buttazzo and G. Dal Maso, Dirichlet problem for demicoercive functionals, Nonlinear Anal. 10 (1986), no. 6, 603-613.

[4] J.M. Ball, B. Kirchheim and J. Kristensen, Regularity of quasiconvex envelopes, Calc. Var. Partial Differential Equations 11 (2000), no. 4, 333-359.

[5] L. Boccardo and B. Dacorogna, Coercivity of integrals versus coercivity of integrands, J. Math. Anal. Appl. 189 (1995), 607-616.

[6] C.-Y. Chen, Existence and global higher integrability of quasiminimizers among minimizing sequences of variational integrals, Malaysian J. Math. Sci. 10(S) (2016), 85-100.

[7] B. Dacorogna, Quasiconvexity and relaxation of nonconvex problems in the calculus of variations, J. Funct. Anal. 46 (1982), no. 1, 102-118.

[8] B. Dacorogna, Direct methods in the calculus of variations. Second edition, Applied Mathematical Sciences, 78, Springer, New York, 2008.

[9] G. Dolzmann, B. Kirchheim and J. Kristensen, Conditions for equality of hulls in the calculus of variations, Arch. Ration. Mech. Anal. 154 (2000), no. 2, 93-100. 
[10] I. Ekeland and R. Temam, Convex analysis and variational problems, Classics In Applied Mathematics, SIAM 28, 1999.

[11] L.C. Evans and R.F. Gariepy, Measure theory and fine properties of functions, Studies in Advaned Mathematics, CRC Press 1992.

[12] I. Fonseca and J. Malý, Relaxation of multiple integrals below the growth exponent, Ann. Inst. H. Poincaré Anal. Non Linéaire 14 (1997), no. 3, 309-338.

[13] I. Fonseca and S. Müller, Quasi-convex integrands and lower semicontinuity in $L^{1}$, SIAM J. Math. Anal. 23 (1992), no. 5, 10811098.

[14] N. Fusco, Quasiconvexity and semicontinuity for higher-order multiple integrals, Richerche Mat. 29 (1980), no. 2, 307-322.

[15] T. Iwaniec, Nonlinear Cauchy-Riemann operators in $\mathbb{R}^{n}$, Trans. Amer. Math. Soc. 354 (2002), no. 5, 1961-1995.

[16] T. Iwaniec and A. Lutoborski, Integral estimates for null Lagrangians, Arch. Ration. Mech. Anal. 125 (1993), no. 1, 25-79.

[17] T. Iwaniec and G. Martin, Geometric function theory and nonlinear analysis, Oxford Mathematical Monographs, The Clarendon Press, Oxford University Press, New York, 2001.

[18] T. Iwaniec and G. Martin, Quasiregular mappings in even dimensions, Acta Math. 170 (1993), no. 1, 29-81.

[19] T. Iwaniec and C. Sbordone, Weak minima of variational integrals, J. Reine Angew. Math. 454 (1994), 143-161.

[20] D. Kinderlehrer and P. Pedregal, Characterizations of Young measures generated by gradients, Arch. Ration. Mech. Anal. 115 (1991), no. 4, 329-365.

[21] B. Kirchheim and J. Kristensen, On rank one convex functions that are homogeneous of degree one, Arch. Ration. Mech. Anal. 221 (2016), no. 1, 527-558.

[22] J. Kristensen, Lower semicontinuity of quasi-convex integrals in $B V$, Calc. Var. PDE 7 (1998), no. 3, 249-261.

[23] J. Kristensen, Lower semicontinuity in spaces of weakly differentiable functions, Math. Ann. 313 (1999), no. 4, 653-710.

[24] J. Kristensen and G. Mingione, The singular set of minima of integral functionals, Arch. Ration. Mech. Anal. 180 (2006), no. 3, 331-398. 
[25] J. Kristensen and F. Rindler, Relaxation of signed integral functionals in BV, Calc. Var. PDE 37 (2010), no. 1-2, 29-62.

[26] P. Marcellini, On the definition and the lower semicontinuity of certain quasiconvex integrals, Ann. Inst. H. Poincaré Anal. Non Linéaire 3 (1986), no. 5, 391-409.

[27] V.P. Maz'ya, Sobolev spaces with applications to elliptic partial differential equations. Second, revised and augmented edition, Grundlehren der Mathematischen Wissenschaften, 342, Springer, Heidelberg, 2011.

[28] N.G. Meyers, Quasi-convexity and lower semi-continuity of multiple variational integrals of any order, Trans. Amer. Math. Soc. 119 (1965), 125-149.

[29] G. Mingione, Regularity of minima: an invitation to the dark side of the calculus of variations, Appl. Math. 51 (2006), no. 4, 355426.

[30] C.B. Morrey Jr., Quasi-convexity and the lower semicontinuity of multiple integrals, Pacific J. Math. 2 (1952), 25-53.

[31] S. Müller, Variational models for microstructure and phase transitions. Calculus of variations and geometric evolution problems (Cetrao, 1996), 85-210, Lecture Notes in Math., 1713, Springer, Berlin, 1999.

[32] S. Müller, V. Šverák and B. Yan, Sharp stability results for almost conformal maps in even dimensions, J. Geom. Anal. 9 (1999), no. $4,671-681$.

[33] P. Rabier, A necessary and sufficient condition for the coerciveness of a class of functionals and its applications, SIAM J. Math. Anal. 15 (1984), no. 2, 367-388.

[34] P. Soneji, Lower semicontinuity in BV of quasiconvex integrals with subquadratic growth, ESAIM Control Optim. Calc. Var. 19 (2013), no. 2, 555-573.

[35] T. Schmidt, Regularity of minimizers of $W^{1, p}$-quasiconvex integrals with $(p, q)$ growth, Calc. Var. Partial Differential Equations 32 (2008), no. 1, 1-24.

[36] E.M. Stein and G. Weiss, Introduction to Fourier analysis on Euclidean spaces, Princeton University Press, 1971.

[37] D. Vasiliu and B. Yan, On a restricted weak lower semicontinuity for smooth functionals on Sobolev spaces, Indiana Univ. Math. J. 55 (2006), no. 2, 869-894. 
[38] B. Yan and Z. Zhou, $L^{p}$-mean coercivity, regularity and relaxation in the calculus of variations, Nonlinear Anal. 46 (2001), 835-851.

[39] W.P. Ziemer, Weakly Differentiable Functions. Sobolev Spaces and Functions of Bounded Variation, Graduate Texts in Math. 120, Springer-Verlag, New York, 1989.

[40] K. Zhang, On various semiconvex hulls in the calculus of variations, Calc. Var. PDE 6 (1998), 143-160.

[41] K. Zhang, On coercivity and regularity for linear elliptic systems, Calc. Var. Partial Differential Equations 40 (2011), 65-97.

[42] K. Zhang, Private communication, (1997).

Department of Mathematics, Faculty of Science, Universiti Putra Malaysia e-mail: cychen@upm.edu.my

Mathematical Institute, Andrew Wiles Building, University of Oxford, Oxford OX2 6GG, U.K.

e-mail: kristens@maths.ox.ac.uk 